POLIWODA, Guido N., Aus Katastrophen lernen. Sachsen im Kampf gegen die Fluten der Elbe 1784 bis 1845

\title{
François Walter
}

\section{(2) OpenEdition}

\section{Journals}

Édition électronique

URL : http://journals.openedition.org/ifha/525

DOI : 10.4000/ifha.525

ISSN : 2198-8943

\section{Éditeur}

IFRA - Institut franco-allemand (sciences historiques et sociales)

Référence électronique

François Walter, «POLIWODA, Guido N., Aus Katastrophen lernen. Sachsen im Kampf gegen die Fluten der Elbe 1784 bis 1845 », Revue de l'IFHA [En ligne], Date de recension, mis en ligne le 01 janvier 2007, consulté le 22 septembre 2020. URL : http://journals.openedition.org/ifha/525 ; DOI : https://doi.org/ 10.4000/ifha. 525

Ce document a été généré automatiquement le 22 septembre 2020.

(CIFHA 


\title{
POLIWODA, Guido N., Aus
} Katastrophen lernen. Sachsen im Kampf
gegen die Fluten der Elbe 1784 bis 1845

\author{
François Walter
}

1 C'est bien évidemment une réflexion sur les pluies torrentielles et les inondations exceptionnelles de l'été 2002 avec leurs conséquences tragiques en Saxe, République tchèque et Autriche qui sert de prologue. À Dresde, l'Elbe a atteint alors un niveau record de 9,40 mètres, ce qui n'était jamais arrivé dans l'histoire ! Même si G.N.P. avait choisi son champ d'investigation avant les événements, il ne peut faire l'impasse sur les analogies supposées avec le passé. C'est à un cycle d'une durée de soixante ans auquel il s'est plus particulièrement intéressé, à savoir les années 1784-1845, marquées - la différence avec aujourd'hui étant fondamentale - par une phase de refroidissement. Les catastrophes dont il s'agit ici sont donc en très grande majorité des inondations hivernales (de novembre à avril), dont la première se manifeste en mars 1784, avec la débâcle rapide des glaces, faisant suite à un hiver particulièrement froid. L'objet de la recherche est de comprendre la manière dont la société globale, au tournant du XIXe s., a réagi à une fréquence accrue d'épisodes catastrophiques, sans perdre bien sûr de vue l'intérêt actuel pour une situation similaire, celle de la répétitivité programmée des crues de l'Elbe, compte tenu du phénomène plus général du réchauffement climatique. L'hypothèse qui traverse le livre est de distinguer déjà dans le passé un changement de paradigme qui pousse les communautés concernées par les inondations à développer non plus simplement des stratégies de protection contre les catastrophes naturelles mais à expérimenter une véritable gestion des risques (Risikomanagement). Dans cette dissertation qui se situe à l'articulation entre histoire de l'environnement et histoire de la gestion des risques naturels, l'auteur analyse surtout la capacité de réaction aux crises. Ce champ de recherche en plein développement s'inspire largement des études pionnières menées, dès le début des années 1990, par Manfred Jakubowski-Tiessen, dont le livre sur l'onde de tempête de 1717 en mer du Nord constitue le modèle. Dans cette perspective, la recherche monographique de G.N.P. apporte une nouvelle contribution à la connaissance de ce qu'il est convenu d'appeler dans le champ 
germanophone "Katastrophenkultur » mais que nous préférons nommer en français les " cultures du risque ", soit autant de situations sociales où les collectivités s'efforcent de réduire l'incertitude liée au retour périodique des calamités. D'où cette thématique du Lernprozess qui figure dans le sous-titre, à savoir comment les sociétés tirent parti de leur expérience de la catastrophe.

2 Le livre est divisé en trois parties. La première explique le déroulement des inondations de 1784, leurs conséquences matérielles et les premières mesures de précaution appliquées ensuite lors du retour d'épisodes semblables, à quatre reprises jusqu'en 1799. La deuxième partie explore la période 1800-1820 durant laquelle s'expérimentent, à l'échelle locale, une véritable gestion des conséquences des hautes eaux et, à l'échelle régionale, la mise en place de mesures administratives destinées à coordonner la prévention et améliorer la réactivité aux situations extrêmes. La troisième partie est celle où la société civile admet un état de fait, celui de la récurrence des hautes eaux. Dresde semble se résigner à vivre une situation «à la vénitienne » et s'organise pour s'y accommoder ! En définitive, la thèse de doctorat de G.N.P. met surtout en évidence la capacité nouvelle de tous les acteurs à développer des compétences réactives à la catastrophe, à les mettre en réseau, et non plus d'attendre, plutôt passivement comme dans le passé, l'impulsion d'une autorité administrative supérieure. La période étudiée serait, du point de vue de ce processus d'apprentissage, exemplaire d'un changement de paradigme. La comparaison inévitable avec la situation actuelle dans le bassin de l'Elbe s'arrête, quant à elle, à des analogies formelles, la nécessité de vivre avec une menace récurrente d'épisodes extrêmes étant la principale d'entre elles. Toujours tentante, l'actualisation instrumentale d'un savoir historique demeure un exercice périlleux dont l'auteur se tire très honorablement.

François WALTER (Université de Genève) 